\title{
Reducing the pain of childhood vaccination: an evidence- based clinical practice guideline (summary)
}

\author{
Anna Taddio BScPhm PhD, Mary Appleton BA, Robert Bortolussi MD, Christine Chambers PhD, \\ Vinita Dubey MD, Scott Halperin MD, Anita Hanrahan RN, Moshe Ipp MD, Donna Lockett PhD, \\ Noni MacDonald MD, Deana Midmer RN EdD, Patricia Mousmanis MD, Valerie Palda MD MSc, \\ Karen Pielak RN MSc, Rebecca Pillai Riddell PhD, Michael Rieder MD PhD, Jeffrey Scott MD, \\ Vibhuti Shah MD MSc
}

Previously published at www.cmaj.ca

See also the complete guideline article by Taddio and colleagues at www.cmaj.ca

I njections for vaccinations, the most common source of iatrogenic pain in childhood, ${ }^{1}$ are administered at multiple times to almost all Canadian children throughout infancy, childhood and adolescence. ${ }^{2}$ The pain associated with such injections is a source of distress for children, their parents and those administering the injections. If not addressed, this pain can lead to preprocedural anxiety in the future, fear of needles and nonadherence with vaccination schedules. ${ }^{3}$ It is estimated that up to $25 \%$ of adults have a fear of needles, ${ }^{4}$ with most fears developing in childhood. ${ }^{5}$ About $10 \%$ of the population avoids vaccination and other needle procedures because of needle fears. ${ }^{3}$

Conversely, minimizing pain during childhood vaccination can help to prevent distress, development of needle fears and subsequent health care avoidance behaviours such as nonadherence with vaccination schedules. More positive experiences during vaccine injections also maintain and promote trust in health care providers. ${ }^{3}$

In light of the prevalence of pain during vaccine injections and the potential for substantial adverse sequelae, we identified a need for a national guidance document to address this important public health issue. Although the topic was covered in a previous narrative review ${ }^{1}$ and a national guideline, ${ }^{4}$ neither of those documents was based on the requisite systematic approach and rigorous guideline development process. Moreover, additional data have been published since the appearance of the previous documents.

Our objective was to develop a clinical practice guideline, based on systematic reviews of the literature, as interpreted by experts, to assist clinicians in managing procedure-related pain and distress among children undergoing vaccine injections. The scope was limited to acute (immediate) pain and distress at the time of vaccine injection in children 0 to 18 years of age. We did not consider the management of delayed-onset pain occurring in the hours or days after the injection. The term "distress" is often used to refer to the combination of pain and anxiety or fear experienced by children before and during painful medical procedures. For the purposes of this guideline, we considered distress and pain together, referring to the combination as "pain."

\section{Key points}

- Vaccine injections performed in childhood are a substantial source of distress.

- Untreated pain can have long-term consequences, including preprocedural anxiety, hyperalgesia, needle fears and avoidance of health care.

- Simple, cost-effective, evidence-based pain-relieving strategies are available.

- A "3-P" approach, combining pharmacologic, physical and psychological factors, improves pain relief.

\section{Methods}

We convened an interdisciplinary expert panel, the Help ELiminate Pain in KIDS (HELPinKIDS) Team, to develop the guideline. Individual panel members, with the required expertise in areas of vaccination and immunization, pediatrics, pain, evidence-based medicine, education, knowledge translation, health policy and guideline development, were

Faculty of Pharmacy (Taddio) and Departments of Paediatrics (Ipp), of Family and Community Medicine (Midmer) and of Health Policy, Management and Evaluation (Palda, Shah), Faculty of Medicine, University of Toronto, Toronto, Ont.; Research Institute (Taddio, Ipp, Pillai Riddell) and Department of Psychiatry (Pillai Riddell), The Hospital for Sick Children, Toronto, Ont.; Canadian Center for Vaccinology (Appleton, Halperin, MacDonald, Scott), Halifax, NS; Departments of Pediatrics (Bortolussi, Halperin, MacDonald, Scott) and of Psychology (Chambers), IWK Health Centre, Halifax, NS; Infectious Diseases and Immunization Committee (Bortolussi) and Drug Therapy and Hazardous Products Committee (Rieder), Canadian Paediatric Society; Departments of Microbiology and Immunology (Bortolussi, Halperin), of Pediatrics (MacDonald) and of Emergency Medicine (Scott), Faculty of Medicine, and Department of Pediatrics/Psychology (Chambers), Faculty of Science, Dalhousie University, Halifax, NS; Toronto Public Health (Dubey), Toronto, Ont.; Capital Health Region (Hanrahan), Edmonton, Alta.; National Advisory Committee on Immunization (Hanrahan); private practice (Lockett), Milton, Ont.; Healthy Child Development Program (Mousmanis), Ontario College of Family Physicians, Toronto, Ont.; Department of Medicine (Palda), St. Michael's Hospital, Toronto, Ont.; British Columbia Centre for Disease Control (Pielak), Vancouver, BC; Department of Psychology (Pillai Riddell), York University, Toronto, Ont.; Children's Hospital of Western Ontario (Rieder), London, Ont.; Department of Medicine (Rieder), University of Western Ontario, London, Ont.; Department of Paediatrics (Shah), Mount Sinai Hospital, Toronto, Ont.

CMAJ 2010. DOI:10.1503/cmaj.092048 
selected from across Canada to represent different perspectives and experiences.

The guideline development process was based on published methods. ${ }^{6}$ Details of the methods can be found in the full guideline (see Appendix 1, available at www.cmaj.ca/cgi/content/full /cmaj.092048/DC1). Briefly, we used published literature, interviews with key informants and discussions with panel members and stakeholder partners, ${ }^{8}$ including parents, ${ }^{8,9}$ to identify 32 clinical questions for consideration in the guideline. We subsequently reduced the number of clinical questions to 18 , to reflect the evidence base, as described below.

Pain management is usually based on a "3-P" approach involving pharmacologic, physical and psychological strategies. Therefore, our evidence base encompassed all of these domains. For the purposes of this guideline, selected panel members performed three systematic reviews and meta-analyses, one for each of the three domains of pain management. ${ }^{10-12}$ We limited the evidence to randomized controlled trials and studies with quasi-experimental designs. We used the Cochrane Risk of Bias Tool to determine the quality of included studies. We critically appraised the evidence and generated recommendations using the evidence-based methods outlined by the Canadian Task Force on Preventive Health Care, ${ }^{13}$ including an accompanying level of evidence and grade for each recommendation (Table 1). ${ }^{14}$

In total, we evaluated 71 studies that included 8050 chil-

Table 1: Criteria for evaluating evidence and grading recommendations*

Level or grade Criteria

\section{Evidence}

I

II-1

II-2

III

\section{Recommendation}

A

B

C

D

E

I

*Adapted, with permission, from Palda and colleagues. ${ }^{14}$ dren. We formulated a recommendation for each clinical question according to the strength of the scientific evidence (i.e., study design and methodologic quality), with consideration of the values that expert reviewers attributed to various outcomes and parents' preferences. We used a consensus process to arrive at the final wording for each recommendation.

We circulated the guideline to relevant experts, some of whom represented stakeholder organizations, for external review according to the AGREE instrument (Appraisal of Guidelines for Research and Evaluation; www.agreetrust .org). In addition, three trained members of the Guidelines Advisory Committee (www.gacguidelines.ca) who were not involved in the guideline development process independently evaluated the guideline. We discussed the comments of all reviewers and incorporated them into the final manuscript, as appropriate. We then finalized and approved the guideline.

Funding for this project was provided by the Canadian Institutes of Health Research. The funding agency had no role in developing the recommendations, and its views and interests did not influence the recommendations.

\section{Clinical recommendations}

For 14 of the 18 questions, there was sufficient evidence to make a practice recommendation to reduce pain (Table 2). These recommendations have been organized into five clusters: infants, injection procedure, parentled strategies, pharmacotherapy and psychological strategies. For the remaining four clinical questions, there was insufficient evidence to make a practice recommendation (Table 3).

Several of the practice recommendations relating to the injection procedure can be implemented immediately by health care providers in all vaccination practice settings, as they do not require planning or additional resources (e.g, time, supplies or money). Examples of these easily adopted pain-relieving strategies include performing intramuscular injections rapidly without prior aspiration, positioning children upright, injecting the most painful vaccine last when multiple vaccines are being administered and providing tactile stimulation.

Performing intramuscular injections rapidly without prior aspiration probably reduces pain through the combined effects of shortening the time of contact between needle and tissue and reducing lateral movement of the needle ("wiggle") within the tissue. The long-standing practice of aspiration was initially proposed to reduce the risk of intravascular injection of the vaccine. However, the sites commonly used for vaccine injections are devoid of large blood vessels, 
Table 2: Recommendations to reduce pain during vaccine injections in children* (part 1 of 2)

$\begin{array}{lcc}\text { Clinical question } & \text { Level of } & \text { Grade of } \\ \text { evidencet recommendationt }\end{array}$

\section{Infants}

Among infants undergoing vaccination, does breastfeeding during the procedure reduce pain at the time of injection?

Among infants undergoing vaccination, does administration of sweet-tasting solutions reduce pain at the time of injection?

\section{Injection procedure}

Among children undergoing vaccination, does administering one commercial brand of a vaccine rather than another commercial brand of the same vaccine cause less pain at the time of injection?

Among children undergoing vaccination, does positioning the child in a supine position result in more pain at the time of injection?

\section{Among children undergoing}

intramuscular injection of vaccine, should slow injection with aspiration be avoided to reduce pain at the time of injection?

Among children receiving multiple vaccine injections at a single vaccination visit, does injecting the most painful vaccine last decrease pain at the time of injection?

Among children undergoing vaccination, does rubbing the skin near the injection site before and during the procedure result in less pain at the time of injection?

\section{Parent-led interventions}

Among children undergoing vaccination, does use of (1) parent-led distraction or (2) parent coaching result in less pain and pain-related distress at the time of injection?

\section{Pharmacotherapy}

Among children receiving intramuscular and subcutaneous injection of vaccines, does application of topical anesthetics on the skin before the injection reduce pain at the time of injection?

\section{Psychological interventions}

Among children undergoing vaccination, does use of clinician-led distraction result in less pain at the time of injection?

Among children undergoing vaccination, does use of child-led distraction result in less pain at the time of injection?
To reduce pain at the time of injection, I breastfeed their infants during vaccination

To reduce pain at the time of injection among infants up to 12 months of age who cannot be breastfed during vaccination, administer a sweet-tasting solution during vaccination

If more than one commercial brand of a vaccine is available, and the brands are interchangeable, inject the least painful brand during vaccination of children, to reduce pain at the time of injection

To reduce pain at the time of injection, do not place children in a supine position during vaccination

To reduce pain at the time of injection, administer intramuscular vaccines to children using a rapid injection technique without aspiration

When administering multiple vaccine injections to children sequentially, inject the most painful vaccine last to reduce pain at the time of injection

To reduce pain at the time of injection among children aged $\geq 4$ years, offer to rub or stroke the skin near the injection site with moderate intensity before and during vaccination

Although there is insufficient evidence for or against the use of parent-led distraction or parent coaching during vaccination of children as a way to reduce pain at the time of injection, clinicians may offer this intervention to parents to reduce pain-related distress

To reduce pain at the time of injection, encourage parents to use topical anesthetics during vaccination of children

To reduce pain at the time of injection, use clinician-led distraction techniques during vaccination of children

To reduce pain at the time of injection among children aged $\geq 3$ years, use childled distraction techniques during vaccination 
Table 2: Recommendations to reduce pain during vaccine injections in children* (part 2 of 2)

\begin{tabular}{|c|c|c|c|}
\hline Clinical question & Recommendation & $\begin{array}{l}\text { Level of } \\
\text { evidence } t\end{array}$ & $\begin{array}{l}\text { Grade of } \\
\text { recommendationt }\end{array}$ \\
\hline $\begin{array}{l}\text { Among children undergoing vaccination, } \\
\text { does slow, deep breathing or blowing } \\
\text { performed by the child result in less pain } \\
\text { at the time of injection? }\end{array}$ & $\begin{array}{l}\text { To reduce pain at the time of injection, } \\
\text { have children aged } \geq 3 \text { years engage in } \\
\text { slow, deep breathing or blowing during } \\
\text { vaccinations }\end{array}$ & 1 & B \\
\hline $\begin{array}{l}\text { Among children undergoing vaccination, } \\
\text { does use of combined psychological } \\
\text { interventions (i.e., interventions that } \\
\text { include at least one cognitive and one } \\
\text { behavioural intervention) result in less } \\
\text { pain and distress at the time of injection? }\end{array}$ & $\begin{array}{l}\text { To reduce pain at the time of injection } \\
\text { among children aged } \geq 3 \text { years, use } \\
\text { combined psychological interventions } \\
\text { during vaccination }\end{array}$ & I & B \\
\hline $\begin{array}{l}\text { Among children undergoing vaccination, } \\
\text { does suggesting that "it won't hurt" } \\
\text { result in less pain at the time of injection? }\end{array}$ & $\begin{array}{l}\text { Do not tell children that "it won't hurt," } \\
\text { as this type of statement, when used } \\
\text { alone, has been shown to be ineffective } \\
\text { in reducing pain at the time of injection }\end{array}$ & I & D \\
\hline
\end{tabular}

*There is some evidence that combining strategies improves pain relief.

thevels of evidence and grades of recommendation are defined in Table 1

and aspiration is no longer regarded as necessary. About onethird of vaccinators do not perform aspiration, and there have been no documented harms caused by omitting this step.

Positioning children upright or holding them during vaccine injections, rather than laying them supine, reduces pain. Children can be seated or held by a parent in a position that is most comfortable for them (e.g., held in a bear hug or on the parent's lap). Although the exact mechanism underlying the reduction in pain associated with this positioning is unknown, it may involve a reduction in anxiety, which in turn reduces the perception of pain. Conversely, excessive restraint may increase the child's distress, so parents and health care providers are encouraged to hold and support children without using excessive force.

Children routinely receive two or more vaccine injections during the same visit. Administering the most painful vaccine last minimizes the priming effect of the first injection on subsequent injections, thus reducing overall pain. There is limited research, however, regarding the optimal order of injection for vaccines that are coupled for administration at the same visit. At present, the two vaccines known to be relatively more painful are M-M-R-II (Merck) and Prevnar (Wyeth). When these are coupled with other vaccines, they should be given last.

Providing tactile stimulation by rubbing or stroking the skin near the injection site before and during vaccine injections reduces pain in children aged four years and older. The proposed mechanism involves blocking the transmission of the pain sensation to the brain by means of competing touch sensation. This technique is often referred to as generating "white noise." The optimal method for rubbing (in terms of frequency, intensity and pattern) is unknown. It should be tailored to the request and comfort level of the individual child.

A few practice recommendations in this guideline, such as breastfeeding or administration of sugar water (for infants) and application of topical anesthetics and psychological interventions (for children of all ages), require some planning or additional resources, or both, on the part of health care providers and children and their families. Health care providers are encouraged to discuss these additional options with parents and children (as appropriate) and to select the strategies best suited to individual children.

Breastfeeding is considered a combined analgesic intervention because several aspects of breastfeeding (e.g., holding the child, skin-to-skin contact, the sweet-tasting milk and the act of sucking) attenuate pain responses. An adequate latch must be established before the injection. This may take about one minute. If an adequate latch cannot be established before the injection or the infant is not being breastfed, sugar water can be administered instead. The mechanism of action of sugar water, although not fully elucidated, may involve release of endogenous opioids through activation of sweet taste receptors and distraction. Sugar water is easily prepared by mixing a packet of sugar with $10 \mathrm{~mL}$ (two teaspoons) of water and feeding some to the infant a minute or two before the injection. The infant should be monitored for minor adverse events such as choking or gagging.

Topical anesthetics block the transmission of pain signals from peripheral nociceptors. They are effective for vaccines that are administered intramuscularly and subcutaneously. At present, limited evidence indicates that these agents do not interfere with the immunogenicity of the vaccine. Topical anesthetics are available for purchase without a prescription, and parents have indicated a willingness to pay for them to mitigate the pain associated with vaccine injection in their children. However, parents require education about the use of these agents, including the exact site or sites of administration, the duration of application and possible adverse effects. Topical anesthetics must be applied ahead of time, about 20-60 minutes before the injection, depending on the commercial product being applied. The topical anesthetic can be applied upon arrival at the clinic or school (by a parent or a qualified health care professional or delegate) or before departure from home. If multiple vaccines are being injected during the same visit, the topical anesthetic can be applied at two separate sites (e.g., right and left legs). The vaccine or vaccines must be injected 
where the anesthetic has been applied. Health care providers can use a nontoxic marker to outline the area of application. The cost per dose is $\$ 5-\$ 10$. Transient changes in skin colour are common. Systemic toxicity is rare but can occur if the dose or the duration of application is excessive.

Distraction is a psychological intervention that involves directing the child's attention away from the procedure. Distraction led by a health care provider is effective for children of all ages. For children three years of age and older, self-led distraction is also effective. Distraction led by a parent is less effective, possibly because the parent has difficulty providing distraction when he or she is also distressed. The panel recommends, however, that clinicians discuss parent-led interventions, including distraction and coaching, on the grounds that some benefits have been observed in terms of general pain-related distress. In addition, parents may benefit from a formal role, and there may be limited availability of other individuals to deliver such interventions. It is important to ensure that age-appropriate distraction strategies are used and that children are engaged with them. When appropriate, involve the child in planning which distraction strategy will be used and in directing the distraction strategy. Examples of distraction strategies include toys (for infants), bubbles (for toddlers), video games (for school-age children) and music (for adolescents).

Deep (tummy) breathing is another effective psychological intervention that can be used for children three years of age and older. Deep breathing can be facilitated by having the child blow bubbles or spin pinwheels with the breath. These aids also act as distraction strategies.

Pain relief is enhanced when individual pain-relieving strategies are combined. Therefore, health care providers are encouraged to use a mix of strategies to mitigate pain. Parents can be enlisted to help combine and coordinate many of these strategies. In particular, parents can prepare their children, apply topical anesthetics, bring a distraction aid to the appointment, coach the child during deep breathing and hold the child.

Additional details of the practice recommendations, including evidence summaries, references and clinical considerations, can be found in the full guideline (www.cmaj.ca/cgi /doi/10.1503/cmaj.101720).

\section{Implementation of the guideline}

The information contained in this guideline is generalizable to healthy children undergoing injection of vaccines worldwide. We offer the following suggestions to assist in implementing the guideline in various settings.

\section{Context and facilitation}

Organizations and health care providers involved in immunization are encouraged to adopt pain management as an integral component of the vaccination process. Supports should

Table 3: Interventions without sufficient evidence to make a recommendation regarding their use to reduce pain during vaccine injections in children

\begin{tabular}{lc}
\hline Clinical question & Level of \\
Recommendation & $\begin{array}{c}\text { Grade of } \\
\text { evidence* }\end{array}$ \\
\hline
\end{tabular}

\section{Injection procedure}

Among children undergoing vaccination, does (1) application of a vapocoolant spray or (2) application of ice or a $\mathrm{cool} / \mathrm{cold}$ pack on the skin before injection of vaccine reduce pain at the time of injection?

Among children undergoing vaccination, does simultaneous injection by two vaccination providers cause less pain at the time of injection than sequential injections by the same provider?

\section{Route of administration}

Among children undergoing vaccination, for vaccines that can be administered intramuscularly or subcutaneously, does administering the vaccines intramuscularly, rather than subcutaneously, cause less pain at the time of injection?

\section{Pharmacotherapy}

Among children undergoing vaccination, does administration of acetaminophen or ibuprofen before the injection reduce pain at the time of injection?
For children undergoing vaccination, there is insufficient evidence for or against the use of skin-cooling techniques (vapocoolants, ice, cool/cold packs) to reduce pain at the time of injection

For children undergoing vaccination, there is insufficient evidence for or against the use of simultaneous injections rather than sequential injections to reduce pain at the time of injection

For children undergoing vaccination, there is insufficient evidence to recommend for or against the use of a specific route of administration for vaccines that can be administered intramuscularly or subcutaneously to reduce pain at the time of injection

For children undergoing vaccination, there is currently no demonstrated benefit of administering acetaminophen or ibuprofen to reduce pain at the time of injection 
be put in place to facilitate the implementation of these recommendations by health care providers.

\section{Required resources}

Some costs may be incurred by the incorporation of these recommendations into practice, because of required training of staff, required time to practise pain management and expenditures to acquire aids (e.g., bubbles) and resources (e.g., pamphlets for parents and children). For the most part, these costs are relatively modest and may be offset by shorter duration of the procedure (because the child's distress and struggling are lessened) and faster recovery time. Many of the practice recommendations are cost-neutral to parents and the health care system (e.g., rapid intramuscular injection without aspiration).

\section{Setting for vaccinations}

Most of these practice recommendations can be incorporated in many settings without adding any time to the vaccination process (e.g., holding infants, tactile stimulation). Pain-relieving strategies that require additional time (e.g., education and preparation, application of topical anesthetics) can be implemented ahead of time, either at home or upon arrival at the vaccination setting, while the child is waiting to be vaccinated. Parents can be asked to pay a nominal fee to cover the cost of analgesic interventions (e.g., topical anesthetics, bubble solution for blowing, sugar water). Alternatively, analgesic interventions can be provided free of charge.

To date, the guideline has been piloted in an outpatient clinic setting and a public health vaccination setting at a middle school. Feedback received suggests that the strategies are feasible and effective, and that parents and children appreciate efforts made to reduce the children's pain.

\section{Assessment and documentation of pain}

Assessment and documentation of pain during vaccine injections are important aspects of providing quality care. These processes allow determination of the effectiveness of analgesic strategies employed and planning for future vaccine injections.

Adult observers (parents, health care providers or both) can use validated pain assessment techniques to assess injection pain in preverbal children and infants. Verbal children can be asked to self-report their pain using age-appropriate techniques. Health care providers are encouraged to document the strategies used to reduce acute pain at the time of vaccine injection, as well as the child's pain score.

\section{Clinical considerations}

Some judgment about the suitability and feasibility of the recommendations is required, as not all of the recommendations will be appropriate or effective in all situations or for all children. In selecting specific pain-relieving strategies for use in a particular situation, clinicians and parents are advised to consider the analgesic effectiveness of individual modalities, the goals for the child and the preferences of the child, the parents and the clinician.

Health care providers should offer pain-relieving options to parents and children (as appropriate) when they are counselling about other aspects of immunization or well-baby and child care, as parents and children are largely unaware of effective pain-relieving strategies.

No single pain-relieving strategy recommended in this guideline has been demonstrated to reliably reduce pain to zero (i.e., to prevent pain). Clinicians are advised to combine different pain-relieving strategies, as such combinations improve pain relief. ${ }^{12}$ However, combining pain-relieving strategies does not ensure pain-free injections.

\section{Tools to support training and implementation}

We developed a knowledge translation plan to facilitate dissemination and implementation of the guideline. This plan incorporates several educational tools, including a guide to pain management for parents and caregivers (Appendix 1, available at www.cmaj.ca/cgi/content/full/cmaj.092048/DC1), a guide to pain management for health care providers (Appendix 2, available at www.cmaj.ca/cgi/content/full/cmaj.092048/DC1), a tool that health care providers can use to assess and document pain (Appendix 3, available at www.cmaj.ca/cgi/content/full /cmaj.092048/DC1) and a website and educational video for parents and health care providers (available online and freely accessible at www.sickkids.ca/Learning/SpotlightOnLearning /profiles-in-learning/help-eliminate-pain-in-kids/index.html). In addition, several professional organizations (listed at the end of the article) have endorsed or supported this guideline and will assist in its dissemination. This assistance includes online links to the guideline and associated tools and incorporation of recommendations in immunization resources. Educational workshops are being offered to various stakeholder groups.

The guides for parents and health care providers are similar and include information about pain-relieving strategies that are relevant to each user group, as well as information about how to implement them during vaccine injections. The documentation tool allows the health care provider to record information about the vaccine or vaccines administered, the child's age, age-appropriate pain assessment techniques and the child's pain score. The form can be inserted into the child's medical chart and/or given to parents and caregivers. The educational video demonstrates the use of pain-relieving strategies.

\section{Limitations}

The recommendations included in this guideline are limited by the evidence that was available at the time of publication of the three systematic reviews. ${ }^{10-12}$ Certain recommendations have more research support than others. In addition, some of the recommendations are applicable to children of all ages, whereas others apply only to subgroups of children.

For some pain-relieving strategies (e.g., use of sweet-tasting solutions, tactile stimulation), the optimal administration technique and the upper and/or lower age limits for effectiveness could not be determined with confidence from the existing evidence.

Some of the research studies upon which the recommendations are based were limited in terms of the inclusion of children and parents with different demographic characteristics and backgrounds. For instance, children with cognitive impairment or a history of traumatic needle procedures might not 
have been included. The guideline panel did not consider these factors in the recommendations; however, we acknowledge that the experience of pain may be mediated by such factors. Moreover, they may influence the pain-relieving strategies that clinicians, parents and children choose to employ.

Our literature search did not identify studies examining the impact on injection-related pain of the environment or setting in which vaccination was performed (e.g., clinic, school), characteristics of the needle and selected aspects of the injection technique (e.g., gauge, length, angle of injection) or the body region where the vaccine was injected (e.g., arm, thigh). We recommend that future studies examine the effect of these factors on pain at the time of vaccine injection.

For this guideline, we did not consider complementary and alternative medicines, and the published effectiveness of such therapies could be included in future revisions.

\section{Directions for future research}

At present, the optimal pain-relieving regimen for nullifying pain, rather than simply diminishing pain, is unknown. Additional research is recommended to determine which painrelieving regimens reliably prevent pain in children of different ages. New technologies for administering vaccines (e.g., microneedles) and needle-free administration techniques (e.g., nasal sprays) offer alternative ways of preventing pain for which further investigation is also required.

The impact of consistent pain management during injection of vaccines on short-term and long-term outcomes, including the child's pain, satisfaction with the vaccination experience, development of needle fears and adherence with vaccination schedules, has not been evaluated. This is clearly an important topic for future research.

The education of all primary stakeholders involved in childhood immunization, including parents, children and health care providers, is fundamental to any improvements in the delivery of vaccine injections in children. Additional research is planned to determine the impact of the knowledge translation interventions for this guideline.

\section{This article has been peer reviewed.}

Competing interests: Anna Taddio has received a clinical trial grant from Gebauer; has received study drugs for clinical trials from Hawaii Medical, Ferndale Laboratories and Gebauer; and has received honoraria for meeting presentations from Wyeth. Moshe Ipp has received unrestricted research grants from Sanofi and GlaxoSmithKline. He has been a consultant for Wyeth, Merck, GlaxoSmithKline and Sanofi; has received honoraria from Novartis, GlaxoSmithKline and Sanofi; and owns stock in Merck and Pfizer. Donna Lockett and Valerie Palda received consultancy compensation for their role in the development of these guidelines and the preparation of this manuscript. Noni MacDonald has received reimbursement for travel expenses from UNICEF and the World Health Organization related to teaching about vaccine safety. She is also the editor of Paediatrics \& Child Health. Michael Rieder holds a Chair in Paediatric Clinical Pharmacology sponsored by the Canadian Institutes of Health Research and GlaxoSmithKline. Jeffrey Scott received an honorarium from Sanofi Pasteur for participation in a session at the 2008 Canadian National Immunization Conference. Vibhuti Shah has received study drugs for clinical trials from Hawaii Medical and Ferndale Laboratories. None declared for other authors.

Noni MacDonald is the Section Editor, Population and Public Health for $C M A J$ and was not involved in the editorial decision-making process for this article.
Contributors: Anna Taddio, chair of the HELPinKIDS Team, was the overall project leader. Anna Taddio, Mary Appleton, Robert Bortolussi, Christine Chambers, Vinita Dubey, Scott Halperin, Moshe Ipp, Donna Lockett, Patricia Mousmanis, Michael Rieder and Vibhuti Shah were responsible for conception of the guideline. Anna Taddio, Christine Chambers and Vibhuti Shah led acquisition of data from the literature, drafted the clinical questions for consideration in the guideline and led the systematic reviews and meta-analyses of the literature. All authors participated in refining the clinical questions, interpreting the data and drafting the recommendations. Anna Taddio wrote the first draft of the manuscript; all authors critically revised the article for important intellectual content and gave final approval of the version to be published.

Endorsements: This guideline has been endorsed by the Canadian Center for Vaccinology, the Canadian Coalition for Immunization Awareness and Promotion, the Canadian Family Practice Nurses Association, the Canadian Nursing Coalition for Immunization, the Canadian Paediatric Society, the Canadian Pain Society, the Canadian Pharmacists Association, the Canadian Psychological Association, and the Community and Hospital Infection Control Association - Canada.

Acknowledgements: The authors thank the family physicians and pediatricans of the Joint Action Committee on Child and Adolescent Health, the College of Family Physicians of Canada and the British Columbia Centre for Disease Control who provided expert review before submission. See also Appendix 4, available at www.cmaj.ca/cgi/content/fullcmaj.092048/DC1.

Funding: Funding for this project was provided through a knowledge synthesis grant (KRS-91783) awarded by the Canadian Institutes of Health Research. The views and interests of the funding agency did not influence the recommendations. Publication of the full guideline was funded by the following agencies: Canadian Institutes of Health Research, Canadian Pain Society, the Linden Fund, BC Ministry of Healthy Living and Sport, Children's Health Research Foundation and the Canadian Center for Vaccinology.

\section{REFERENCES}

1. Schechter NL, Zempsky WT, Cohen LL, et al. Pain reduction during pediatric immunizations: evidence-based review and recommendations. Pediatrics 2007;119:e1184-98.

2. Immunization schedules: recommendations from the National Advisory Committee on Immunization (NACI). Ottawa (ON): Public Health Agency of Canada. Available: www.phac-aspc.gc.ca/im/is-cv/index-eng.php\#a (accessed 2009 Jan. 9).

3. Taddio A, Chambers CT, Halperin SA, et al. Inadequate pain management during childhood immunizations: the nerve of it. Clin Ther 2009;31(Suppl 2):S152-67.

4. Guideline statement: management of procedure-related pain in children and adolescents. J Paediatr Child Health 2006;42(Suppl 1):S1-29.

5. Hamilton JG. Needle phobia: a neglected diagnosis. J Fam Pract 1995;41:169-75.

6. Harris RP, Helfand M, Woolf SH, et al. Current methods of the US Preventive Services Task Force: a review of the process. Am J Prev Med 2001;20(3 Suppl):21-35.

7. Taddio A, Manley J, Potash L, et al. Routine immunization practices: use of topical anesthetics and oral analgesics. Pediatrics 2007;120:e637-43.

8. Taddio A. Proceedings of the workshop conquering pain: the hidden cost of immunization; 2008 Jan. 28; Toronto. Toronto (ON): University of Toronto; 2008 Available: http://resources.cpha.ca/CCIAP/data/700e.pdf (accessed 2009 Jan. 25).

9. Parvez E, Stinson J, Boon H, et al. Mothers' beliefs about analgesia during childhood immunization. Paediatr Child Health (Oxford) 2010;15:289-93.

10. Taddio A, Ilersich AL, Ipp M, et al.; HELPinKIDS Team. Physical interventions and injection techniques for reducing injection pain during routine childhood immunizations: systematic review of randomized controlled trials and quasirandomized controlled trials. Clin Ther 2009;31(Suppl 2):S48-76.

11. Chambers CT, Taddio A, Uman LS, et al.; HELPinKIDS Team. Psychologica interventions for reducing pain and distress during routine childhood immunizations: a systematic review. Clin Ther 2009;31(Suppl 2):S77-103.

12. Shah V, Taddio A, Rieder MJ; HELPinKIDS Team. Effectiveness and tolerability of pharmacologic and combined interventions for reducing injection pain during routine childhood immunizations: systematic review and meta-analyses. Clin Ther 2009;31(Suppl 2):S104-47.

13. Canadian Task Force on Preventive Health Care. New grades for recommendations from the Canadian Task Force on Preventive Health Care. CMAJ 2003;169. 207-8.

14. Palda VA, Guise JM, Wathen CN; Canadian Task Force on Preventive Health Care. Interventions to promote breast-feeding: applying the evidence in clinical practice. CMAJ 2004;170:976-8

Correspondence to: Dr. Anna Taddio, Leslie L. Dan Faculty of Pharmacy, University of Toronto, 144 College St., Toronto ON

M5S 3M2; anna.taddio@utoronto.ca 